\title{
sciendo
}

\section{Analysis of Scoring Sequences in Matches of the Portuguese Premier League}

\author{
by \\ José M. Pratas ${ }^{1}$, Anna Volossovitch ${ }^{1}$, Ana I. Carita ${ }^{2}$
}

\begin{abstract}
The aim of this study was to examine the sequences of the first two goals scored in soccer matches in accordance with a range of different match contexts. Data from 1506 matches played in the Portuguese Premier League during six consecutive competitive seasons (2009-10 to 2014-2015) were analysed using descriptive statistics and the chi-square test in order to verify the association between variables and a Cox regression analysis was used to predict the time the second goal was scored in function of the time of the first goal scored in the match and the scoreline. The results revealed a higher frequency of the second goals being scored in the second half of a match (58\%) and in the last 5 min periods of each half. A positive association was found for home teams and score-doubling goals (58\%), as well as for away teams and score-equalizing goals (56\%). For home and away teams the score-doubling goal of a match was strongly and positively associated with a win outcome for home (93\%) and away teams (92\%), while the scoreequalizing goals were associated with a draw (home and away teams: $44 \%$ ) and loss outcome (home: $33 \%$ and away teams: $32 \%)$. Finally, the Cox model showed that if the first goal was scored in the second half of the match, the probability of the second goal being scored was three times higher compared to the first half.
\end{abstract}

Key words: game analysis, soccer, survival analysis.

\section{Introduction}

In soccer, it has been demonstrated that performance of teams can be influenced by the scoreline (Lago-Peñas, 2012; Gómez et al., 2013). Soccer players perform significantly less highintensity activity when winning than when losing or when the score is tied (Lago et al., 2010). It was also shown that teams had longer periods of possession in matches when they were losing than when they were winning (Lago-Peñas and Dellal, 2010; Lago-Peñas and Gomez-Lopez, 2014), teams played more in the attack and defensive zones when the score was level (Lago, 2009) and finalthird entries were greater when teams were 1 goal down than when they were 1 goal up (Lago-Peñas and Gomez-Lopez, 2014). Findings also showed that shots on goal decreased by $14.1 \%$ and $14.45 \%$ when teams were 1 goal up and when the scores were level, respectively (Lago-Peñas and GomezLopez, 2014) and when a team was drawing or winning, the probability of reaching the goal decreased by 43 and $53 \%$, respectively (LagoBallestero et al., 2012).

The score evolution in time is an important situational factor that may influence the team performance during the match and it also allows to identify the critical moments of the game (Leite, 2013; Njororai, 2014). In low scoring games, each goal can be considered a critical incident that influences the course of the game (Ferreira, 2013). Studies conducted with different professional soccer leagues have shown that the team which scores first in a match has a higher probability of winning, and the home team is more likely to score first than the opposing team

\footnotetext{
1 - CIPER, Faculdade de Motricidade Humana, SpertLab, Universidade de Lisboa, Portugal.

2 - CIPER, Faculdade de Motricidade Humana, BIOLAD, Universidade de Lisboa, Portugal, Estrada da Costa, 1495-688 Cruz Quebrada, Portugal.
} 
(Molinuevo and Bermejo, 2012; Tenga, 2012; Pratas et al., 2016). Most of these studies focused only on the analysis of the first goal effect on the match outcome, and did not examine the role of goals subsequently scored. A recent study of Lago-Peñas et al. (2016) that analysed games played in the most important European domestic leagues (English FA Premier League, French Ligue 1, Spanish La Liga, Italian Serie A and German Bundesliga) in the 2014/2015 season demonstrated that home teams scored first in $57.8 \%$ of games and obtained in total $84.85 \%$ of points won in these games. On the contrary, when the away team scored first, they obtained only $76.25 \%$ of subsequent points.

The average number of goals scored per game in each of the major European soccer leagues is not more than three goals per match. Moreover, scores of 2-0 and 1-1 have been two of the five most frequently recorded full-time scores in the 5 best leagues in Europe (England, France, Germany, Italy and Spain) in recent seasons (Anderson and Sally, 2013). Interestingly, in the 2015-16 season, 1-1 was the most common result in these leagues (http://www.soccerstats.com). The same trend was observed in the Portuguese Premier League. In the 2015/16 season, 20\% of matches ended with a total of two goals (i.e. the sum of total goals scored per game by both teams): $10 \%$ finished $1-1$ (home draw, 38 matches), 7\% 2-0 (home win, 21 matches) and 3\% 0-2 (home defeat, 10 matches). The home advantage effect was confirmed in these matches. The home teams of the Portuguese Premier League scored 80 goals, while away teams scored 58 goals.

The advantage of playing at home may be related to several factors reported in literature, such as crowd support (Wolfson et al., 2005), travel (Pollard et al., 2008), familiarity with the game environment (Loughead et al., 2003), referees (Brandão et al., 2011) and territoriality aspects (Sampedro and Prieto, 2011). When the local team scores first, it excites fans and increases their interest in the match, while the away team's early leading may distract the audience from the match (Courneya, 1990). From the sociopsychological point of view, the local public support is considered by own fans as decisive, which can reinforce the self-esteem of players and increase social identity that leads to improvement of team performance and a reduction of the negative effects of stress and anxiety (Wolfson et al., 2005). From the strategic and tactical point of view, scoring first and gaining a score advantage allow the winning team to extend the range of tactical options, for instance by creating more counterattack opportunities against the opposite team, which can opt for more risky strategies in the game.

The second goal might have a decisive impact on the match outcome, which is not less than the influence of the first goal (Anderson and Sally, 2013). The second goal may be the equalizer (0-1 to 1-1, or 1-0 to 1-1) or the goal, which enables a team to double their advantage (1-0 to $2-0$, or $0-1$ to $0-2)$. The anticipation of temporal localization of the second goal associated to its nature (i.e., goal which creates advantage or recovers from score disadvantage) seems to be useful for a timely adaptation of the team's tactics. However, there is a lack of data in the literature to the second goal of a match. One of the few studies which analysed this goal has been conducted by Nevo and Ritov (2012). The interaction between two random goal-scoring times (of the first and second goals) during a soccer match was examined in 760 games played from 2008 to 2010 (two full seasons) in the English Premier League. Using survival analysis methods the authors reported that the first goal occurrence could either expedite or impede the next goal, depending on the time it was scored.

In order to provide a better understanding of which factors influence the second goal being scored, it would be useful to examine how the time of the second goal is associated with a range of different match contexts. A soccer match is a complex dynamic process, in which certain events influence the subsequent course of the match, and this influence must be considered in analysis. In order to explain the relationship between different events, it is necessary to extract information regarding match progress using time as a variable of interest (Venturelli et al., 2011). Several studies have suggested using survival (also known as timeevent) analysis for this purpose (Castilla, 2007; Nevo and Ritov, 2012). This tool involves the use of regression methods for explaining the relationship between independent variables and the time of an event of interest and it is 
considered to be the most suitable means for characterizing and explaining match progress compared to other tools commonly used in soccer performance analysis (Barros et al., 2009; Del Corral et al., 2008).

Thus, the first aim of this study was to analyse the association between the type of the second goal scored in a match (i.e. goal that increased the leading team's advantage or the goal that re-established equality in the scoreline) and the match venue, as well as the final match outcome. Secondly, the study aimed to examine the influence of the time when the first goal was scored and the current scoreline on the probability of the second goal being scored in a match. This knowledge could help coaches anticipate match scenarios and adopt in a timely manner the most appropriate tactics for the remaining part of the match.

\section{Methods}

\section{Sample}

The sample consisted of 1506 matches played in the Portuguese Premier League during six consecutive competitive seasons (from 2009-10 to 2014-2015). All data were collected from the official League website (http://www. ligaportugal.pt).

\section{Statistical Analysis}

Descriptive statistics and chi-square analysis were performed to examine second goals scored during the game period and the association between the type of the second goal being scored (score-doubling or score-equalizing) and the match venue as well as the final match outcome. In $29 \%$ of matches (443 out of 1506), the second goal was not scored. About $9 \%$ of matches (135 out of 1506) ended goalless and in $20 \%$ of matches (308 out of 1506) just one goal was scored. Thus, data were collected from 1063 matches in which the second goal was scored by a home team (586).

A Cox proportional hazards $(\mathrm{PH})$ model was used to estimate the time of the second goal as a function of the time of the first goal in a match and the scoreline.

In survival analysis, the home team was considered as the reference team and the second goal of the match, when it was scored by the home team, was considered as the event of interest. If the away team scored the second goal of the match, this goal was considered as a censored observation. The response variable in the Cox Model was the time elapsed from the time when the first goal in a match was scored to the occurrence of the second goal, considered as the event of interest. Second goals scored in additional time in the first and second halves were recorded at the 45th and 90th min, respectively. Matches in which no goals or only one goal was scored were not considered. Almost half of 1063 matches (477) were censored because the away team scored the second goal of the match.

The Cox model relies on the assumption of the proportionality of hazards, implying that the factors analysed have a constant impact on the hazard over time (Broström, 2012). The proportionality assumption of data was checked to ensure a non-violation of the proportionality assumption. For this purpose the function cox.zph in the survival package, version 2.37-7 was used (Therneau, 2015). The $p$-value obtained in this function was significant $(p<0.05)$ and indicated that the proportionality assumption was not met for the variable time of the first goal in a match. Since the time of the first goal is a continuous covariate, it was necessary to categorize it in order to ensure the proportionality assumption. First, the distribution of the time of first goals was checked using a histogram, which showed that the interval of time during which first goals were scored may reasonably be split into four equallength intervals using the cut function. The time of the first goal in a match is expressed as time in minutes from the start of the match and categorized in accordance with four classes: first period [0-15 $\mathrm{min}]$, second period [16-30 $\mathrm{min}]$, third period [31-45 $\mathrm{min}$ ] and second half [46-90 min]. The current scoreline was also considered as a categorical variable in accordance with two classes: a score-doubling goal (the second goal that doubled the advantage) and an equalizing goal.

All statistical analysis was performed using Software $\mathrm{R}$, version 3.0.2. For all analyses, statistical significance was set at $p<0.05$.

\section{Results}

Data analyses showed that significantly more second goals were scored in the second half of matches $(\chi 2=28.81, p<0.05)$ compared to the 
first half ( $58 \%$ v. $42 \%$, respectively). The highest frequency of the second goal being scored was recorded in the last 5 min periods of each half (Figure 1).

As it can be seen in Table 1, 55\% of second goals were scored by home teams and $45 \%$ by away teams. Home teams presented a greater percentage $(58 \%)$ of second goals that doubled score advantage (1-0 to $2-0$, or $0-1$ to $0-2)$ as compared with away teams (44\%). On the other hand, away teams scored a higher percentage $(56 \%)$ of goals, which restored the score equality (1-0 to $1-1$, or $0-1$ to $1-1$ ) in comparison with home teams $(42 \%)$. The results of the chi-square test $(\chi 2$ $=20.23, p<0.05)$ established a significant association between the match venue and the type of the second goal scored.

Table 2 shows a significant association between the type of the second goal scored and the match outcome $(\chi 2=521.08, p<0.05)$. The team, which scored the second goal, won the match in $59 \%$ of cases. The score-doubling goal was strongly positively associated with a win outcome and the equalizing goals with a draw and loss outcome.

Table 3 displays a statistically significant association between the match outcome and the type of the second goal scored by home teams $(\chi 2=298.85, p<0.05)$ as well as by away teams $(\chi 2$ $=218.55, p<0.05)$.

When home teams scored the scoredoubling goal, they won $93 \%$ of matches, and when they scored the score-equalizing goal, they won $23 \%$, drew $44 \%$ and lost $33 \%$ of matches. Away teams, when they scored the scoredoubling goal, achieved victory in $92 \%$ of matches, and after scoring the equalizing goal they won $24 \%$, drew $44 \%$ and lost $32 \%$ of matches.

Additionally, the frequency of the scoredoubling goal of a match scored by home and away teams was positively associated with a win outcome and negatively associated with draw and loss outcomes. Draw and loss outcomes were significantly and positively associated with the equalizing goal of a match scored by both home and away teams and negatively associated with a win outcome.

The variables estimated using the Cox proportional hazard regression model (Table 4) revealed a significant positive effect of the time of the first goal on the next goal occurrence, i.e. first goal occurrence in later game periods steadily increased the probability of the second goal in a match being scored. At the same time, for home teams the current scoreline had no significant effect on the second goal occurrence in a match.

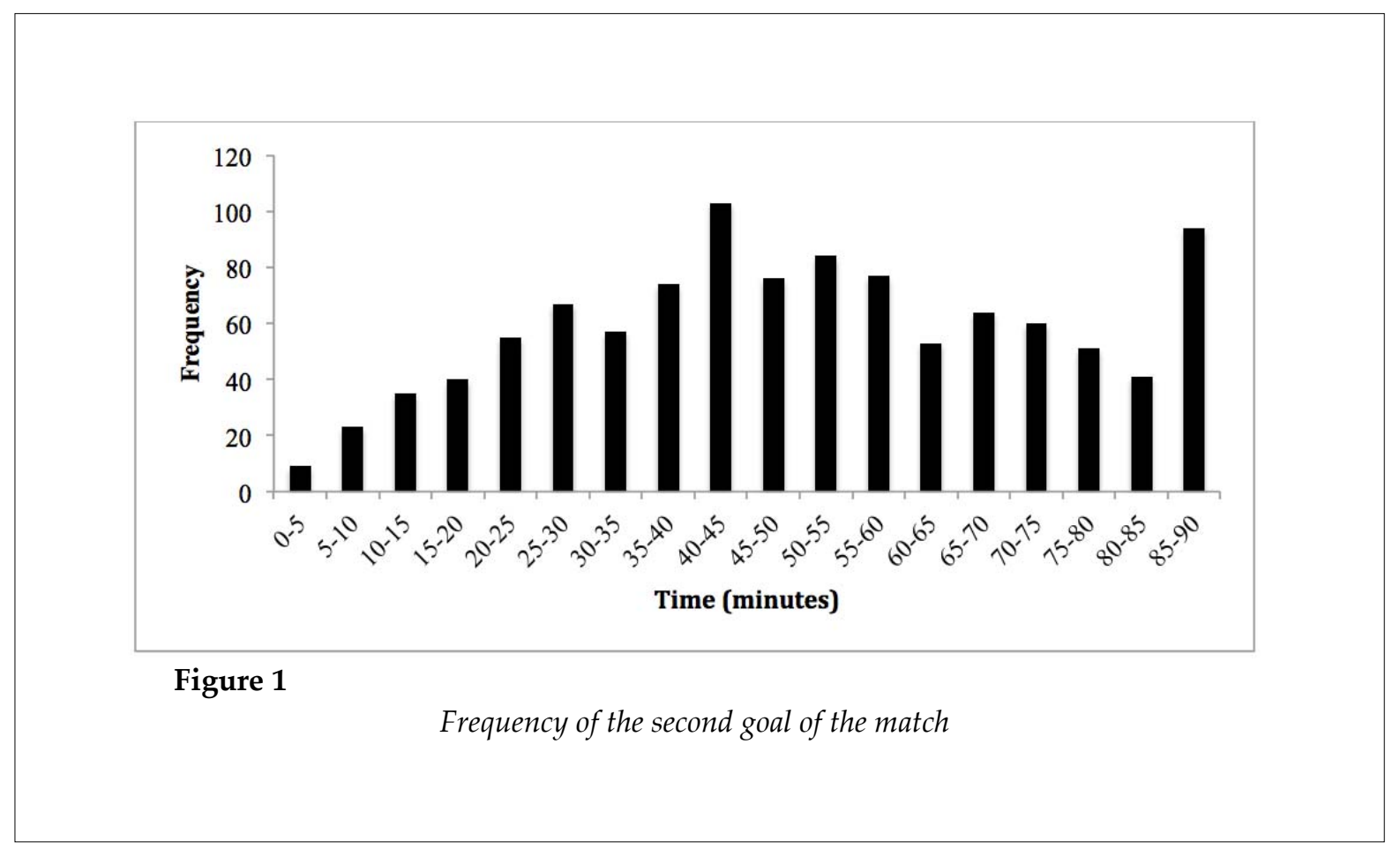


Table 1

Frequency, percentage and chi-square value for the second goal scored by home and away teams cross-referenced with the type of the second goal

\begin{tabular}{|c|c|c|c|c|}
\hline \multicolumn{5}{|c|}{ Type of second goal } \\
\hline Second goal of match & $\begin{array}{c}\text { Score-doubling } \\
1-0 \text { to } 2-0, \text { or } 0-1 \text { to } \\
0-2\end{array}$ & $\begin{array}{l}\text { Score-equalizing } \\
1-0 \text { to } 1-1 \text {, or } 0-1 \text { to } 1-1\end{array}$ & $\mathrm{n}$ & $\chi^{2}$ \\
\hline Home Team & $338(58 \%)^{b}$ & $248(42 \%)^{a}$ & $\begin{array}{c}586 \\
(55 \%)\end{array}$ & \multirow{3}{*}{$20.23^{*}$} \\
\hline \multirow[t]{2}{*}{ Away Team } & \multirow[t]{2}{*}{$209(44 \%)^{a}$} & \multirow[t]{2}{*}{$268(56 \%)^{b}$} & $\begin{array}{r}477 \\
(45 \%) \\
\end{array}$ & \\
\hline & & & 1063 & \\
\hline
\end{tabular}

Statistical significance: ${ }^{*} p<0.05 ;{ }^{a}$ Negative association (adjusted residual $<-1.96$ );

${ }^{b}$ Positive association (adjusted residual $>1.96$ )

Table 2

Frequency, percentage, and chi-square value for the type of the second goal cross-referenced with the outcome of a match

\begin{tabular}{|c|c|c|c|c|c|}
\hline \multirow{2}{*}{$\begin{array}{c}\text { Type of the second } \\
\text { goal }\end{array}$} & \multicolumn{3}{|c|}{ Outcome } & \multirow{2}{*}{ Total } & \multirow{2}{*}{$\chi^{2}$} \\
\hline & Win & Draw & Loss & & \\
\hline \multirow{3}{*}{$\begin{array}{l}\text { Score-doubling } \\
\text { Score-equalizing }\end{array}$} & $506(93 \%)^{b}$ & $33(6 \%)^{a}$ & $8(1 \%)^{a}$ & 547 & \multirow{3}{*}{$521.08^{*}$} \\
\hline & $123(24 \%)^{\mathrm{a}}$ & $225(44 \%)^{\mathrm{b}}$ & $168(32 \%)^{\mathrm{b}}$ & 516 & \\
\hline & $629(59 \%)$ & $258(24 \%)$ & $176(17 \%)$ & 1063 & \\
\hline
\end{tabular}

Statistical significance: ${ }^{*} p<0.05$; ${ }^{a}$ Negative association (adjusted residual $<-1.96$ );

${ }^{b}$ Positive association (adjusted residual $>1.96$ )

Table 3

Frequency, percentage and chi-square value for the type of the second goal scored by home and away teams cross-referenced with the match outcome

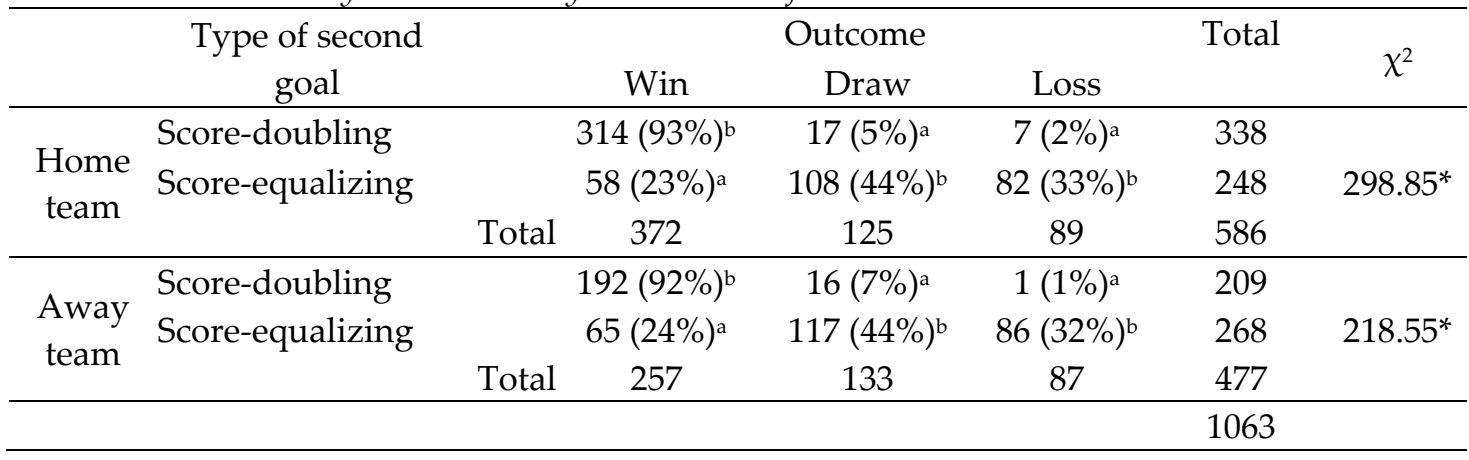

Statistical significance: ${ }^{*} p<0.05 ;{ }^{a}$ Negative association (adjusted residual $<-1.96$ );

${ }^{b}$ Positive association (adjusted residual >1.96) 


\begin{tabular}{|c|c|c|c|c|}
\hline \multicolumn{5}{|c|}{ Cox proportional hazard regression model } \\
\hline Variable & & Coefficient & Hazard Ratio & $p$ \\
\hline \multicolumn{5}{|c|}{ Time of the first goal } \\
\hline & $0-15$ & & 1.00 & \\
\hline & $16-30$ & 0.349 & 1.419 & $0.001 *$ \\
\hline & $31-45$ & 0.645 & 1.906 & $<0.001 *$ \\
\hline & Second half & 1.131 & 3.099 & $<0.001$ * \\
\hline \multicolumn{5}{|l|}{ Scoreline } \\
\hline & Score-equalizing goal & & 1.00 & \\
\hline & Score-doubling goal & 0.0001 & 1.00 & 0.998 \\
\hline \multicolumn{5}{|c|}{ Statistical significance: ${ }^{*} p<0.05$} \\
\hline
\end{tabular}

\section{Discussion}

In the present study, the time of the second goal scored in soccer matches was analysed. As it can be seen in Figure 1, in general, teams scored a larger number of second goals in the second half of matches, and the highest number of goals was scored during the last minutes of each half. Previous studies reported that the frequency of goals scored during a match was time-dependent, more goals were scored in the second half of matches and in the last 10- and 15-min periods of each half (Armatas et al., 2009; Dobson and Goddard, 2010). The tendency for increasing scoring rates over the time of a match could be attributed to physiological and tactical factors that influence teams' performance. On the one hand, fatigue, which is greatest at the end of each half, leads to an increase in the number of technical and tactical errors, which may lead to more goal scoring opportunities. On the other hand, the little time remaining until the end of each half encourages players to use their last chances to score a goal that also may influence the match outcome.

In low-scoring team games such as soccer, scoring the second goal in a match could be crucial for winning. The results of this study confirm the effect of home advantage on the second goal being scored and subsequent victory in matches in the Portuguese professional Football League (Table 1). It was also found that the match venue had an impact on the second type of the goal scored. Score-doubling goals were positively associated with home matches, while equalizing goals were associated with away matches. Courneya and Carrón (1992) and Wolfson et al. (2005) suggested that an early lead in a match caused greater excitement and involvement of fans when the home team scored first. Score advantage reduces the negative effects of stress and anxiety and positively influences team's performance, which could help home teams to enhance their score advantage.

The higher number of equalizing goals scored by away teams may be related to the defensive strategic behaviour adopted by home teams when they are winning and trying to maintain their score advantage. This tendency was reported by Dobson and Goddard (2010) for matches played in the English Premier League and by Lago-Peñas and Gómez-López (2014) for the Spanish Professional League matches, where teams used defensive strategy when winning and 
attacked more when losing in order to maintain the score advantage or to change the current result, respectively. A team that switches from a defensive to attacking style usually increases their own and also opponent's probabilities to score (Armatas et al., 2009; Dobson and Goddard, 2010).

Home and away teams, which scored a doubling goal, won the majority of matches. Additionally, the doubling goals were positively associated with a winning match outcome. Since soccer is a low-scoring team sport, this result was expected. At the same time, the equalizing goals were positively associated with the draw and loss outcome; however, home teams and away teams both avoided defeat in approximately $68 \%$ of matches after scoring an equalizing goal, thus this goal was extremely important.

Regardless of the type of a goal (scoredoubling or equalizing), scoring in soccer always has an impact on the subsequent course of the game, influencing players mentally and frequently leading to tactical adjustments of team's play. Survival analysis demonstrated that the first goal scored in a match had a significant positive effect on the probability of the next goal being scored, and this probability was dependent on time, when the first goal of the match was scored (Table 4). Cox model variables revealed that the probability of the second goal of the match being scored by home teams gradually increased as a match progressed. When the first goal of the match was scored in the second or third 15-min period of the first half, the probability of home teams scoring the second goal increased by $42 \%$ and $190.6 \%$ respectively, as compared with the situation when the first goal of a match was scored in the first 15-min period of the first half.

Moreover, if the first goal occurred only in the second half, the probability of the home team scoring the second goal increased threefold $(309.9 \%)$ as compared with cases where the first goal of the match was scored in the first 15 minutes. A similar tendency was reported for the Spanish Football League by Nevo and Ritov (2012), who suggested that, depending on the time when the first goal was scored, it could accelerate or delay the time when the next goal was scored. When a goal is scored before the 52nd minute, its effect on the probability of the next goal being scored is negative, while when the first goal is scored after that time the effect is positive. Furthermore, the results of the study demonstrate that once a goal is scored, another goal is less likely to be scored as compared with the situation where no goal has been scored (Nevo and Ritov, 2012).

This tendency can be explained by the influence of contextual factors on teams' performance during the match, which has been demonstrated by several studies (Collet, 2013; Pratas et al., 2016). For instance, Heuer and Rubner (2012) showed that during the last ten minutes of matches of the German Premier Football League (Bundesliga) players' behaviour depends significantly on the current score, representing an increasing offensive (or decreasing defensive) behavior (Heuer and Rubner, 2012).

\section{Practical implications}

The findings of the present study could prove useful for soccer coaches, as knowing that the occurrence of the first goal in later match periods steadily increases the probability of the second goal of a match being scored by the home team; therefore, coaches may adjust their decisions related to substitutions or tactical options in terms of attack and defence. During soccer practice players should be prepared for scenarios of mental pressure and physical fatigue, without reducing their self-efficacy in the last periods of training sessions.

\section{Conclusions}

The results of the present study demonstrate that the highest number of second goals (both score-doubling and equalizing) in a match was scored in the last 5-min period of each half. Different types of the second goal (scoredoubling or equalizing) had a different impact on the match outcome for home and away teams. The time of the first goal in a match had a significant effect on the time of the next goal scored by home teams. This paper clearly demonstrates the influence of the match context (i.e. match venue, scoreline, and time of the first goal) on the probability of the second goal of a match being scored and also on the effect of the second goal on the match outcome. Further research is needed to identify performance indicators which are related to the time of the second goal being scored during soccer matches in different European leagues. 


\section{Acknowledgements}

The first author received a PhD grant from the Portuguese Foundation for Science and Technology (SFRH/BD/80719/2011).

\section{References}

Anderson C, Sally D. The numbers game. New York: Penguin Books; 2013

Armatas V, Yiannakos A, Papadopoulou S, Skoufas D. Evaluation of goals scored in top ranking soccer matches: Greek "Superleague" 2006-07. Serbian J Sport Sci, 2009; 3: 39-43

Barros CP, Frick B, Passos J. Coaching for survival: the hazards of head coach careers in the German 'Bundesliga'. Appl Econ, 2009; 41: 3303-3311

Brandão R, Sidónio S, Krebs R, Araújo D, Machado AA. The meaning of refereeing: perception of professional soccer referees. Rev Psicol Deporte, 2011; 20: 275-286

Broström G. Event History Analysis with R. Boca Raton: CRC Press; 2012

Castilla EJ. Dynamic Analysis in the Social Sciences, $1^{\text {st }}$ Edition. USA: Elsevier, Inc; 2007

Collet $\mathrm{C}$. The possession game? A comparative analysis of ball retention and team success in European and international football, 2007-2010. J Sport Sci, 2013; 31: 123-136

Courneya KS. Importance of game location and scoring first in college baseball. Percept Motor Skill, 1990; 71: 624-626

Courneya KS, Carrón AV. The home advantage in sport competitions: A literature review. J Sport Exerc Psychol, 1992; 14: 13-27

Cox DR. Regression models and life tables. J Roy Stat Soc B, 1972; 34: 187-220

Del Corral J, Barros CP, Prieto-Rodríguez J. The Determinants of Soccer Player Substitutions: A Survival Analysis of the Spanish Soccer League. J Sports Econ, 2008; 9: 160-172

Dobson S, Goddard J. Optimizing strategic behaviour in a dynamic setting in professional team sports. Eur J Oper Res, 2010; 205: 661-669

Heuer A, Rubner O. How Does the Past of a Soccer Match Influence Its Future? Concepts and Statistical Analysis. PLoS ONE, 2012; 7

Lago C. The influence of match location, quality of opposition, and match status on possession strategies in professional association football. J Sport Sci, 2009; 27: 1463-1469

Lago C, Casais L, Dominguez E, Sampaio J. The effects of situational variables on distance covered at various speeds in elite soccer. Eur J Sport Sci, 2010; 10: 103-109

Lago-Ballesteros J, Lago-Peñas C, Rey E. The effect of playing tactics and situational variables on achieving score-box possessions in a professional soccer team. J Sport Sci, 2012; 30: 1455-1461

Lago-Peñas C. The role of situational variables in analysing physical performance in soccer. J Hum Kinet, 2012; 35: 89-95

Lago-Peñas C, Dellal A. Ball possession strategies in elite soccer according to the evolution of the matchscore: the influence of situational variables. J Hum Kinet, 2010; 25: 93-100

Lago-Peñas C, Gomez-Lopez M. How important is it to score a goal? The influence of the scoreline on match performance in elite soccer. Percept Motor Skill, 2014; 119: 774-784

Lago-Peñas C, Gómez-Ruano M, Megías-Navarro D, Pollard R. Home advantage in football: Examining the effect of scoring first on game outcome in the five major European leagues. Int J Per An Sport, 2016; 16(2): 411-421

Leite WSS. Analysis of goals in soccer World Cups and the determination of the critical phase of the game. Phys Educ Sport, 2013; 11(3): 247-253

Loughead TM, Carrón AV, Bray SR, Kim A. Facility familiarity and the home advantage in professional sports. Int J Sport Psychol Exerc Psychol, 2003; 1: 264-274 
Molinuevo JS, Bermejo JP. The effect os scoring first and home advantage in professional spanish football and indoor soccer leagues. Rev Psicol Deporte, 2012; 21: 301-8

Nevo R, Ritov Y. Around the goal: Examining the effect of the first goal on the second goal in soccer using survival analysis methods. J Quant An Sports, 2012; 9: 65-177

Njororai W. Timing of Goals Scored in Selected European and South American Soccer Leagues, FIFA and UEFA Tournaments and the Critical Phases of a Game. Int J Sports Sci, 2014; 4(6A): 56-64

Pollard R, da Silva CD, Nísio CM. Home advantage in football in Brazil: Differences between teams and the effect of distance traveled. Brazilian J Soccer Sci, 2008; 1: 3-10

Pratas JM, Volossovitch A, Carita AI. The effect of performance indicators on the time the first goal is scored in football matches. Int J Per An Sports, 2016; 16: 347-54

Sampedro J, Prieto J. The territoriatility as a factor associated to the advantage of playing at home. A comparative study by regions in the Spanish Football League and the in the Spanish Futsal League. Motricidad. Eur J Hum Movement, 2011; 26:93-104

Tenga A. First goal and home advantage at different levels of play in professional soccer. World Congress of Performance Analysis of Sport IX, London \& New York: Routledge Taylor \& Francis Group, Editors: D. Peters, P. G. O’Donoghue, 47-51; 2012

Therneau T. A Package for Survival Analysis in S. R package version 2.37-7, 2015. Available at: http://CRAN.R-project.org/package=survival; accessed on 01.08.2016

Venturelli M, Schena F, Zanolla L, Bishop D. Injury risk factors in young soccer players detected by a multivariate survival model. J Sci Med Sport, 2011; 14: 293-298

Wolfson S, Wakelin D, Lewis M. Football supporters' perceptions of their role in the home advantage. J Sport Sci, 2005; 23: 365-374

\section{Corresponding author:}

\section{José M. Pratas}

Affiliation: CIPER, Faculdade de Motricidade Humana, SpertLab, Universidade de Lisboa

Address: Estrada da Costa, 1495-688 Cruz Quebrada, Lisboa, Portugal

Tel: +351965220648

Fax: + 351214144712

E-mail: jmpratas@fmh.ulisboa.pt 